\title{
Gallstone ileus in the Jordanian Royal Medical Services in a 10-year period
}

S.M. Jarbou' 'and F.A. Al-Hammourl'

SUMMARY Six patients ( 5 women and 1 man, with a median age of 71 years) were operated on for gallstone ileus in the 10-year period 1988-98. The diagnosis was made before operation in 2 patients. The obstructing stones were located in the terminal ileum in 3 patients and in the proximal ileum or jejunum in 3 patients. Choledochoduodenal fistuia was present in all patients. A single-stage procedure (removal of the impacted stone, fistula repair and cholecystectomy) was performed in 1 patient, enterolithotomy alone in 4 patients and resection of part of the intestine where the stone was impacted with primary anastomosis performed in 1 patient. In the postoperative period, 2 patients developed infection of the wound and 2 patients developed deep vein thrombosis; 1 of these died of pulmonary embolism.

\section{Patients}

Between January 1988 and December 1998, 6 patients were treated for gallstone ileus at King Hussein Medical Centre and the Prince Hashem Hospital of the Royal Medical Services in Jordan, representing $1.2 \%$ of 485 patients admitted with mechanical bowel obstruction during this period. The median age of the patients was 71 years (range 62-82 years). A total of 4 patients $(66.6 \%)$ had serious concomitant disease including heart failure, diabetes mellitus, hypertension and ischaemic heart disease, while 2 patients (33.3\%) had a past history of symptomatic gall bladder stones.

\section{Diagnosis and treatment}

The clinical presentation was consistent with bowel obstruction in 5 patients $(83.3 \%)$ and with acute cholecystitis in 1 patient. All the patients had plain abdominal $X$-ray films taken on admission. In 2 of these, the classic signs of gallstone ileus were visible (Figures 1 and 2). In the remaining 4 patients, the films showed signs of bowel obstruction (air fluid levels). Gallstone ileus was diagnosed before operation in 2 patients $(33.3 \%)$. Bowel obstruction was located in the terminal ileum in 3 patients and in the proximal ileum or jejunum in another 3 patients.

A single stone, ranging from $2.8 \mathrm{~cm}$ to $4.0 \mathrm{~cm}$ in length, was found in all cases (Figure 3).

All the patients underwent surgical treatment after stabilization of their medical condition. Enterolithotomy alone was porformed in 4 cases, and small bowel resection of the site of stone impaction in 1 case because of unhealthy hacmorrhagiclooking serosa (Figures 4 and 5). One patient underwent a single-stage procedure in

'Department of General Surgery, King Hussein Medical Centre, Amman, Jordan.

Received: 04/70/9y; accepted: 14/03/00

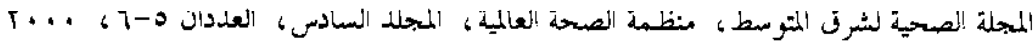


which enterolithotomy, fistula repair and cholecystectomy were performed. There was no operative mortality, but in the postoperative period, 2 patients developed in- fection of the wound and a further 2 patients developed deep vein thrombosis; 1 of these patients died due to pulmonary embolism.

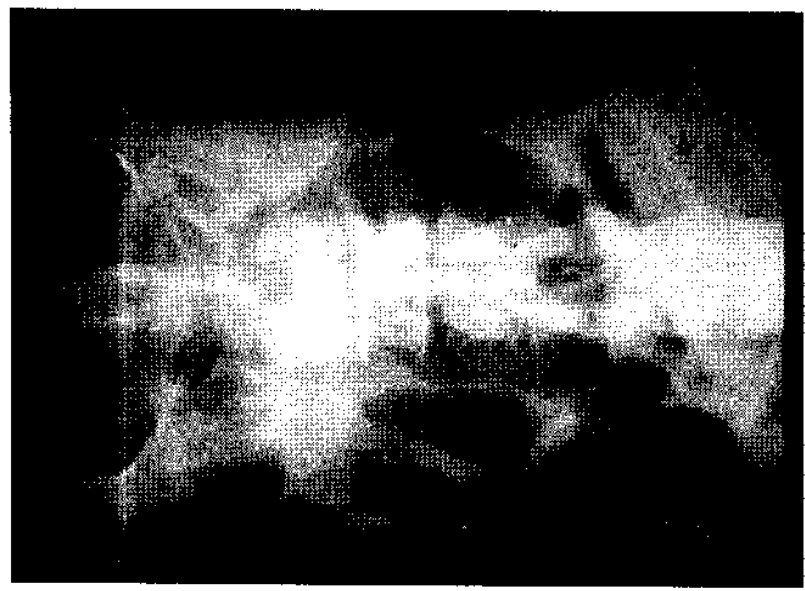

Figure 1 Plain abdominal $X$-ray showing pneumobilia, intestinal obstruction and pelvic stone.

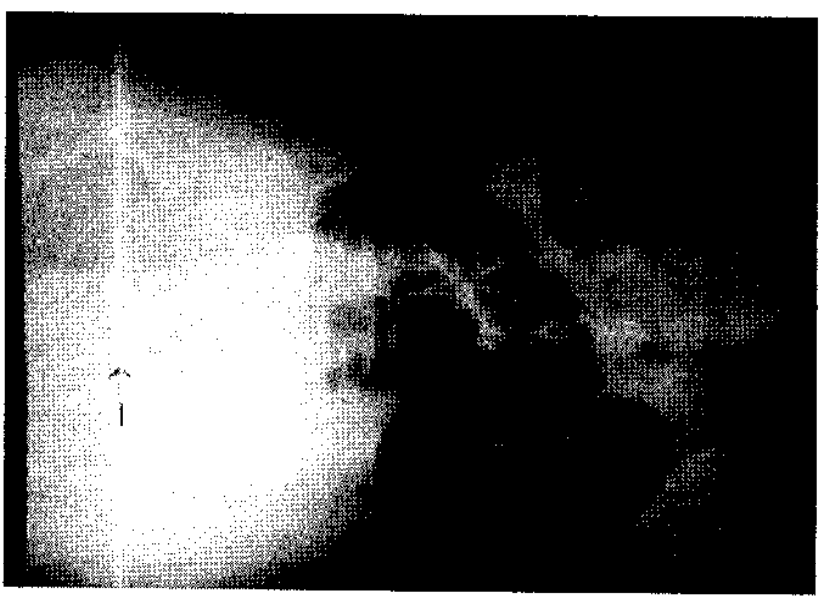

Figure 2 Plain abdominal X-ray showing the same features of gallstone ileus.

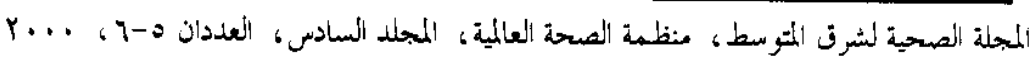




\section{Discussion}

Gallstone ileus was first described by Bartholin in 1654 as a mechanical intraluminal intestinal obstruction caused by impaction of gallstone in the bowel lumen [I]. He considered it a geriatric surgical emergency accounting for $1 \%-4 \%$ of mechanical intestinal obstructions [2].

The stone usuaily gains access to the intestinal tract via a fistula between the gall bladder and adjacent viscera, predominant-

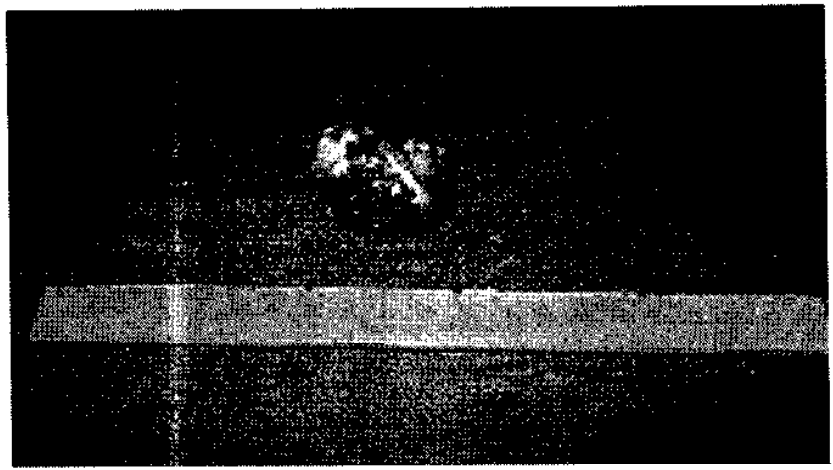

Figure 3 Extracted gallstone measuring $4 \mathrm{~cm}$ in length.

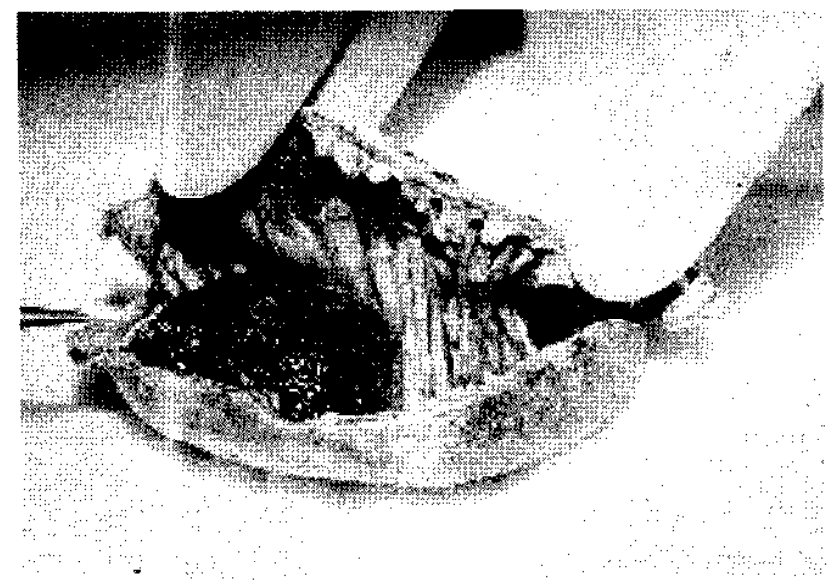

Figure 4 Resected part of small bowel showing unhealthy serosa at site of stone impaction.

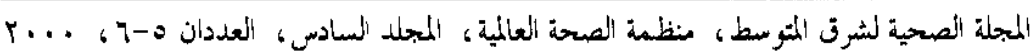




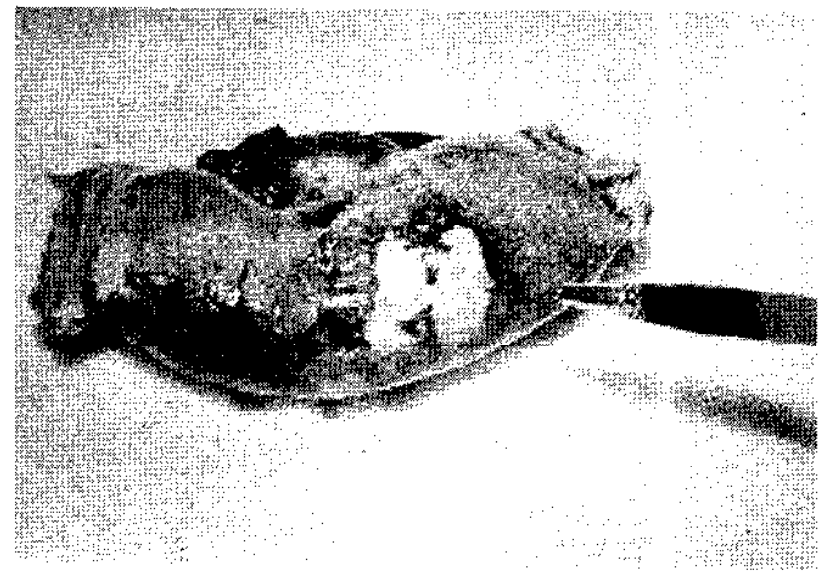

Figure 5 Resected bowel with the stone inside.

ly the duodenum, following acute cholecystitis and adhesions between the gall bladder and adjacent bowel [I].

The disease occurs more frequently in women than in men, in a ratio of 4-8:1 $[2,3]$, and mostly at over 65 years of age [1-4]. The clinical presentation of gallstone ileus is that of intestinal obstruction with abdominal pain, vomiting and abdominal distension in $80 \%$ of patients [2]. Less commonly the presentation is that of acute cholecystitis or jaundice $[2,3]$.

The classic findings on plain abdominal radiographs include: pneumobilia, intestinal obstruction and aberrantly located gallstone (Figures 1 and 2). The presence of two of these findings is considered pathognomonic of gallstone ileus $[2,5,6]$. In general, diagnosis is made before the operation in less than half of patients; in our group this proportion was one-third [7-9]. Once the stone enters the gastrointestinal tract, it may be vomited, passed spontaneously or become impacted, most frequently in the terminal ileum, which is the narrowest part of small bowel [I]. The size of the obstructing stone ranges from 2.5 to $5 \mathrm{~cm}$ $[7,10]$. Stones of less than $2.5 \mathrm{~cm}$ in length are usually passed spontaneously.

The management of gallstone ileus is controversial. The choice is between performing simple enterolithotomy with longitudinal incision on the antimesenteric border proximal to the site of impaction (bowel resection is sometimes necessary if there is perforation, as in 1 of our patients), or a single-stage procedure involving enterolithotomy, cholecystectomy and fistula closure. Most studies suggest that simple enterolithotomy is appropriate in the majority of patients because it is relatively simple and minimizes operative time for high-risk patients, while the one-stage procedure is indicated in patients at low risk and when local and general conditions permit $[2,6,11]$.

\section{Conclusion}

Gallstone ileus is a rare and serious geriatric emergency. It is not a common cause of 
mechanical bowel obstruction. The appropriate surgical procedure should be select- ed to treat the obstruction and save the life of the patient.

\section{References}

1. Van Landingham SB, Broders CW. Gallstone ileus. Surgical clinics of North America. 1982, 62:241-7.

2. Clavien PA et al. Gallstone ileus. British journal of surgeny, 1990, 77:737-42.

3. Moss JF et al. Gallstone ileus. American surgeon, 1987, 53:424-8.

4. Davies JB, Sedman PC. Benson EA. Gallstone ileus - beware the silent second stone. Postgraduate medical journal, 1996, 72(847):300-1.

5. Van Hillo $M$ et al. Gallstone obstruction of the intestine. An analysis of ten patients and a review of literature. Surgery, 1987. 101:273-6.

6. Deitz DM et al. Improving the outcome in gallstone ileus. American journal of ourgery, 1986, 151:572-6.
7. Kasahara $Y$ et al. Gallstone ileus, The American journal of surgery, 1980, 145: 437-40.

8. Kurtz RJ et al. Patterns of treatment of galistone ileus over a 45-year period. American journal of gastroenterology, 1985, 80:95-8.

9. Svartholm $E$ et al. Diagnosis and treatment of gallstone ileus. Acta chirurgica scandinavica, 1982, 148:453-8.

10. Jenkins HP, Evans R, Kollert W. Gallstone ileus. Surgical clinics of North America, 1961, 41:71-81.

11. Rodríguez-Sanjuán JC et al. Cholecystectomy and fistula closure versus enterolithotomy alone in gallstone ileus. British journal of surgery, 1997, 84(5): 634-7. 